Musées, Patrimoine et Culture scientifiques et techniques

$144 \mid 2012$

novembre - décembre 2012

\title{
Le musée Exploramer : à la découverte du milieu marin du Saint-Laurent
}

\section{Sandra Gauthier}

\section{OpenEdition \\ Journals}

Édition électronique

URL : http://journals.openedition.org/ocim/1136

DOI : 10.4000/ocim. 1136

ISSN : 2108-646X

Éditeur

OCIM

Édition imprimée

Date de publication : 1 novembre 2012

Pagination : 48-51

ISSN : 0994-1908

Référence électronique

Sandra Gauthier, « Le musée Exploramer : à la découverte du milieu marin du Saint-Laurent », La Lettre de l'OCIM [En ligne], 144 | 2012, mis en ligne le 01 novembre 2014, consulté le 20 avril 2019. URL : http://journals.openedition.org/ocim/1136; DOI : 10.4000/ocim.1136 


\section{Le musée Exploramer : à la découverte du milieu marin du Saint-Laurent}

\section{Sandra Gauthier *}

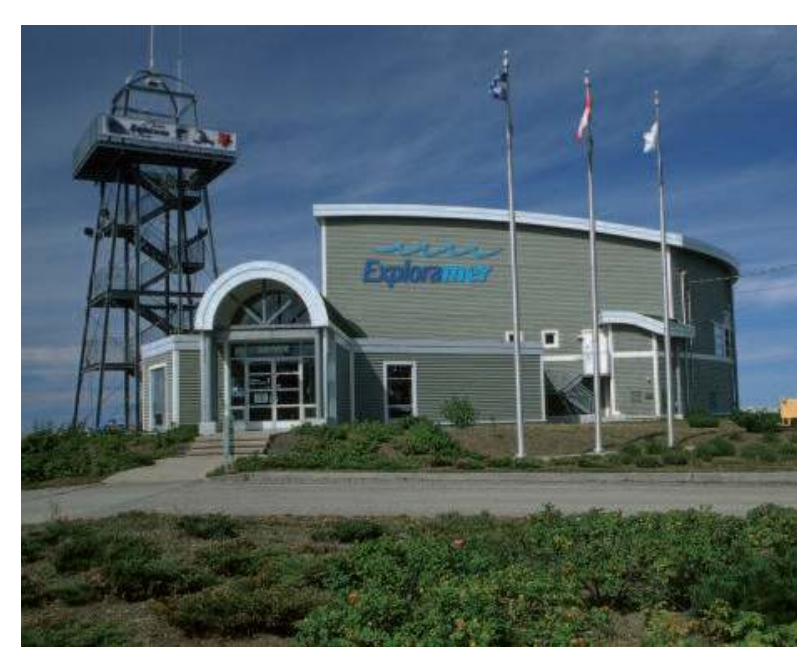

Le site d'Exploramer (C) Exploramer
* Sandra Gauthier est directrice générale d'Exploramer à Sainte-Anne-des-Monts (Québec) sandra.gauthier@exploramer.qc.ca
Organisé autour de la muséologie et des sciences marines, Exploramer a pour principale mission de sensibiliser les publics à la préservation et à la reconnaissance du milieu marin du Saint-Laurent dans l'environnement à travers des activités de vulgarisation scientifique proposées au musée mais également lors d'excursions en mer.

À Sainte-Anne-des-Monts, ville du littoral nord de la péninsule gaspésienne au Québec, la mer définit le quotidien de sa population. En fait, Sainte-Anne-desMonts est située à la limite de l'estuaire et du golfe du Saint-Laurent. Ce grand fleuve prend sa source dans les Grands Lacs, au cœur de l'Amérique du Nord, pour se jeter dans l'océan Atlantique par le Détroit de Cabot entre Terre-Neuve et la Nouvelle-Écosse.

L'équipe du musée Exploramer y anime une institution entièrement dédiée à la découverte et à la préservation des habitats et des espèces marines du Saint-Laurent, avec pour objectif principal de partager ses connaissances auprès d'un large public. En plus d'offrir une exposition permanente dédiée aux ressources marines du Saint-Laurent, l'équipe d'Exploramer offre aux visiteurs la possibilité de se rendre en mer sur un bateau conçu pour la découverte du monde marin.

\section{En vedette : les bassins tactiles}

La tournée des bassins de ce parc aquarium permet aux visiteurs de toucher et de manipuler une vingtaine d'espèces de crustacés, mollusques et échinodermes 


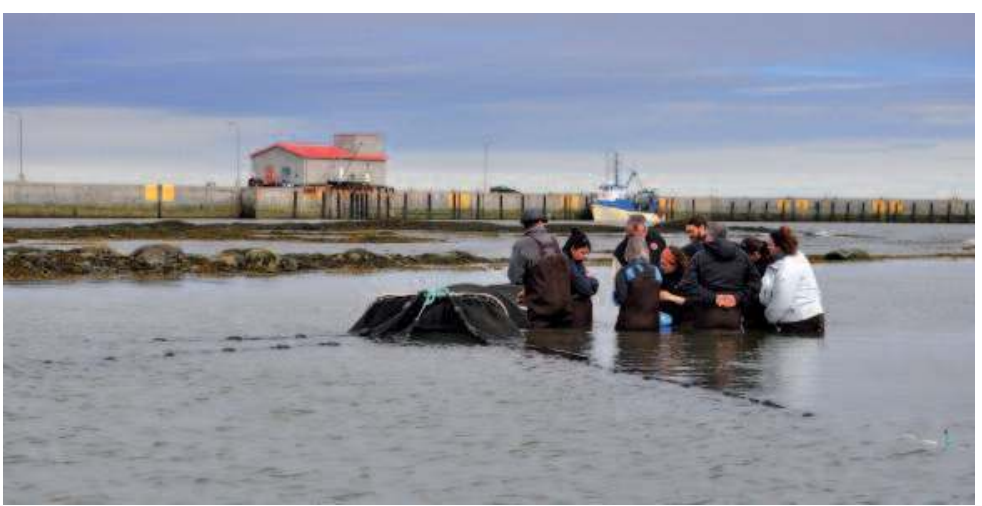

Exploramer propose aux visiteurs de participer à la cueillette de données scientifiques dans les eaux du Saint-Laurent.

(C) Exploramer/Pascal Lévesque

vivants. L'aménagement de cet espace pédagogique éveille les sens des visiteurs tout en piquant leur curiosité. Les homards, crabes, bernard-l'hermite, oursins, concombres de mer, étoiles de mer polaires et différents mollusques peuvent être manipulés avec soin, sous la supervision de guides-naturalistes. Les bassins tactiles sont ainsi devenus une marque de commerce d'Exploramer. On y retrouve aussi une quinzaine d'aquariums et de bassins qui composent l'exposition permanente Un jardin sous la mer. On y présente une collection vivante constituée d'environ quatre-vingts espèces marines totalisant plus de 800 individus dont le loup atlantique, la raie tachetée, l'oursin vert, le crabe commun et l'anémone plumeuse. L'ensemble traite de la biodiversité du golfe et de l'estuaire du Saint-Laurent en fonction des profondeurs marines et de leur relief.

Le musée propose notamment l'exposition Yeux ouverts dans la mer, où l'on observe les fonds marins par la lunette d'un scaphandrier. Aussi, afin de comparer les cinq sens traditionnels de l'Homme avec ceux des mammifères marins, l'équipe a conçu l'exposition La mer dans tous les sens. Celle-ci suggère, entre autres, de tester le goût chez le phoque ou la vision complètement séparée des côtés droit et gauche de la tête du cachalot. Un exercice pour le moins déconcertant. Également, l'équipe a voulu favoriser un réel contact de ses visiteurs avec le milieu marin et ainsi leur faire apprivoiser la vie avec la mer omniprésente. Des excursions écologiques sont ainsi proposées à bord du navire JV Exploramer. Parallèlement, selon le rythme des marées, une activité de cueillette de poissons à l'aide d'un verveux (filet de pêche en forme d'entonnoir) se fait les pieds dans l'eau, à marée basse, près de la rive et du quai.

\section{Fourchette bleue et Guide de consommation écoresponsable}

Il est apparu logique à l'équipe d'Exploramer d'inciter le public à goûter, et ainsi apprécier à leur juste valeur, les diverses espèces de poissons et de crustacés peuplant la zone côtière. Pour cela Exploramer a créé le label Fourchette bleue, un programme de certification des restaurants et poissonneries qui choisissent de s'investir dans une démarche favorisant une saine utilisation des ressources marines. À SainteAnne-des-Monts, les pêcheurs sont les voisins d'Exploramer. L'institution a donc souhaité développer un concept de développement durable où l'industrie de la pêche devient un acteur de la préservation du Saint-Laurent et non pas un secteur économique montré du doigt comme un simple exploiteur de la ressource. Après avoir vu certains concepts mis de l'avant par d'autres aquariums tels que ceux de Vancouver ou de Boston, où les produits de la mer les plus populaires sont différenciés selon des feux : rouge (espèces à ne pas consommer), jaune (à consommer rarement) et vert (sans restriction), Exploramer a plutôt opté pour l'idée de « déguster autre chose ». Avec cette nouvelle étiquette Fourchette blene, associée au développement durable, les pêcheurs peuvent maintenant diversifier leurs prises en ramenant au quai des espèces de poissons et invertébrés autrefois rejetées à la mer parce qu'elles ne trouvaient pas preneur. Les pêcheurs, distributeurs et restaurateurs se disent enthousiastes de l'élan créé par ce programme favorisant la mise en valeur de poissons,

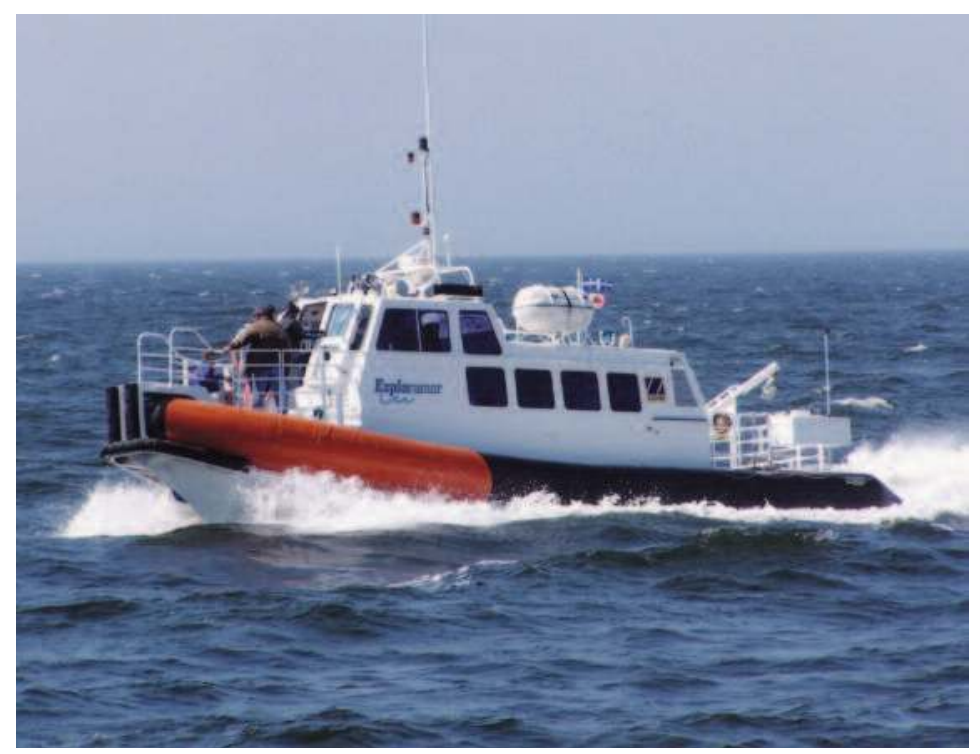

Des excursions en bateau qui permettent de découvrir la faune du Saint-Laurent. (c) Exploramer 
crustacés, mollusques et échinodermes méconnus. Pour les grands chefs, inscrits dorénavant au firmament des «rock stars du petit écran », ces nouvelles espèces représentent des défis culinaires forts intéressants. Ils peuvent maintenant apprêter par exemple l'hémitriptère, le chaboisseau, le crabe araignée, le crabe commun ou la mactre de Stimpson.

Exploramer s'est également associé au Biodôme de Montréal dans le cadre d'un projet de développement d'un guide écoresponsable de la consommation des produits de la mer. En effet, alors que tout nous rappelle qu'il faut faire des choix responsables pour aider notre planète, le consommateur se sent parfois coupable quand il va acheter du saumon d'élevage ou du thon rouge à la poissonnerie. La consommation de poisson est bénéfique pour la santé humaine : or, de nos jours, on généralise au point où l'acte de manger du poisson est souvent synonyme d'un appui à la dégradation de notre environnement et à des mises en garde liées à leur contamination. Afin d'aider le consommateur dans ses choix, il existe une multitude de programmes de sensibilisation sur les produits marins. Ils prennent généralement la forme de petits feuillets éducatifs qui se glissent dans la poche et qui fournissent une liste de choix à faire aux abords des étalages des poissonniers. La cause est juste : elle fait appel à l'urgence d'agir pour que l'industrie mondiale des pêches se conforme aux principes de conservation des populations de poissons démontrant des indices d'épuisement.

Mais dresser une liste c'est forcément exclure des espèces ou des secteurs de cette industrie. Ces feuillets sont-ils trop alarmistes ou biaisés ? Manquentils leur cible ? L'équipe du Biodôme de Montréal s'est penchée sur les grands principes et la rigueur scientifique des méthodes d'évaluation disponibles au Canada afin de mesurer la pertinence de ces outils éducatifs. Elle a conclu que la base de ces outils s'appuie sur une méthodologie qui semble rigoureuse, mais ces guides font souvent abstraction de notions de proximité de la ressource et de la présence d'une industrie canadienne des pêches et de l'aquaculture respectueuses de l'environnement et des avis et règlements.

Au grand étonnement des spécialistes du Biodôme, ces listes sont souvent élaborées à l'extérieur des frontières canadiennes ou calquées sur des outils décisionnels déjà bien implantés. Elles préconisent des espèces non-indigènes et elles ne considèrent que très peu les

\section{Deux reconnaissances pour des actions concrètes de participation citoyenne}

En 2010, le musée Exploramer de Sainte-Anne-desMonts reçoit le prix québécois de l'entreprise citoyenne, dans la catégorie secteur coopératif, pour son programme incitatif Fourchette bleue. En faisant de la sensibilisation et de l'éducation auprès de ses publics, Exploramer amorce ainsi une réflexion sur les actions concrètes à entreprendre, par les citoyens, pour contribuer à préserver la biodiversité dans le fleuve Saint-Laurent. Ce programme novateur passe à l'action en valorisant, par l'intermédiaire des restaurants et poissonneries, des espèces marines méconnues et non menacées.

Voici la liste officielle des espèces de l'année 2012 recommandée par l'équipe de scientifiques du musée : Baudroie d'Amérique, Bigorneau, Buccin commun (bourgots), Calmar du Saint-Laurent, Caplan, Chaboisseau sp., Crabe araignée, Crabe commun, Flétan Atlantique, Flétan du Groenland (turbot), Hémitriptère Atlantique, Huître (aquaculture), Laminaire, Mactre de Stimpson, Maquereau bleu, Moule bleue (aquaculture), Mye commune (aquaculture), Oursin vert, Pétoncle géant (aquaculture), Phoque du Groenland.
Il s'agit ainsi de diversifier la consommation de produits et de réduire la pression de pêche sur les espèces en difficulté. Au lieu de dresser une liste rouge d'espèces à ne pas consommer, Fourchette bleue revalorise d'autres espèces à découvrir. Une stratégie aussi astucieuse qu'éthique, qui respecte autant l'environnement que le travail des pêcheurs.

Puis en 2012, Exploramer est sélectionné pour rejoindre les rangs de la Collection d'expériences distinctives (CED) de la Commission canadienne du tourisme pour l'activité Un laboratoire sur la mer. Une excursion en mer permet, aux visiteurs du musée, de participer à la cueillette de données scientifiques et à la remontée des casiers de pêche. Puis, à l'aide d'instruments scientifiques, les visiteurs observent le monde de l'infiniment petit à l'infiniment grand, du plancton aux baleines. Les membres de la Collection d'expériences distinctives sont choisis pour la qualité et l'originalité de l'expérience touristique qu'ils proposent. 
aspects socio-économiques liés aux pêcheries et à l'aquaculture comme des activités de développement durable et moteurs économiques des régions côtières.

Le Biodôme de Montréal, en collaboration avec Exploramer, est donc en train d'élaborer un guide écoresponsable pour la consommation de poissons marins et de fruits de mer pour l'Est du Canada. Un guide sans liste! Il favorisera l'aspect éducatif touchant l'exploitation des ressources marines de manière durable et responsable et la responsabilisation du consommateur. Ce guide misera sur le jugement des consommateurs et abordera les grands concepts liés à l'acquisition de comportement écoresponsables dans les habitudes de consommation. En attendant la version finale de ce guide, le meilleur geste à poser est de questionner son poissonnier, car plus il y aura de réponses, plus le consommateur pourra faire des choix sensés : on parlera alors de traçabilité et d'une approche holistique.

\section{En mission 12 mois sur 12}

Avec le temps, l'équipe d'Exploramer a conçu des activités pédagogiques extra-muros, à l'instar d'Explo-Mer. Il s'agit d'un concours inspiré des " expos-sciences » qui s'adresse au premier cycle du cours secondaire dans les écoles des régions du Bas-Saint-Laurent et de la Gaspésie toutes deux situées sur le territoire maritime du Québec. L'ensemble des activités liées à ExploMer s'intègre au cours d'initiation aux sciences et à la technologie. Un chapitre du programme pédagogique de ce cours est dévolu à l'étude de la biodiversité du Saint-Laurent. Ainsi, les biologistes d'Exploramer présentent en classe toute une portion consacrée à la

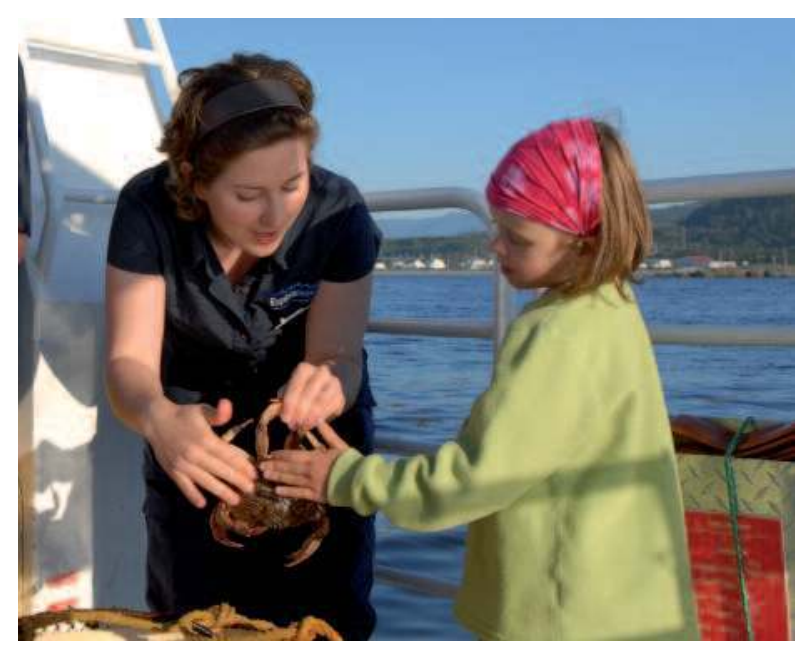

Animation autour du Tourteau (C) Exploramer biologie marine, sous ses aspects académiques et didactiques. Les guides-naturalistes enrichissent leurs présentations avec l'usage de bassins tactiles portatifs. Les élèves doivent, à leur tour, faire une recherche scientifique sur une espèce qu'ils ont eux-mêmes choisi d'étudier plus à fond. Ils auront ensuite à répondre à la problématique suivante : comment votre espèce survivra-t-elle aux changements climatiques ?

\section{La clef de la réussite : les guides-naturalistes}

Les guides-naturalistes, dans les diverses activités proposées, s'illustrent comme de véritables protagonistes, spécialistes de la biodiversité des habitats marins du Saint-Laurent, qui vulgarisent toute l'information pertinente. Les visites guidées et les activités tactiles représentent le cœur de la programmation d'interprétation d'Exploramer. Les excursions en mer s'effectuent trois fois quotidiennement et la cueillette de poissons dans le verveux se réalise au moins une fois chaque jour.

Aujourd'hui, Exploramer semble avoir trouvé sa vitesse de croisière. D'autres projets mijotent dont celui de développer une nouvelle exposition sur les requins du Saint-Laurent. L'équipe d'Exploramer demeure convaincue de la nécessité d'une meilleure transmission des connaissances pour favoriser une plus grande sensibilisation à la fragilité de l'écosystème marin du Saint-Laurent.

\section{Bibliographie}

Adey, W-H. et Loveland, K. Dynamic Aquaria : Building Living Ecosystems. San Diego : Academic press, 2007, 528 p.

Fontaine, P-H. La faune sous-marine du Saint-Laurent. Québec. Éditions Multimondes, 2005, 227 p.

Colt, J. et Huguenin, J-E. Design and Operating Guide for Aquaculture Seawater Systems, Developments in Aquaculture and Fisheries Science, vol. 33. Seattle et Woodshole : Éditions Elsevier, 2002, 328 p.

Scott, M-G. et Scott, W-B. Atlantic Fishes of Canada. Toronto : Presses de l'université de Toronto, 1998, 730 p.

Pêche impact, le journal des pêches, périodique bimestriel, Grande-Rivière (Québec), Impression Acadie Presse (www.pecheimpact.com)

www.exploramer.qc.ca 